Association for Information Systems AIS Electronic Library (AISeL)

Wirtschaftsinformatik Proceedings 2003

Wirtschaftsinformatik

September 2003

\title{
Etablierung von Wissensgemeinschaften in virtuellen Organisationen
}

Volkmar Pipek

Universität Siegen, pipek@cs.uni-bonn.de

Markus Won

Universität Bonn

Follow this and additional works at: http://aisel.aisnet.org/wi2003

\section{Recommended Citation}

Pipek, Volkmar and Won, Markus, "Etablierung von Wissensgemeinschaften in virtuellen Organisationen" (2003). Wirtschaftsinformatik Proceedings 2003. 54.

http://aisel.aisnet.org/wi2003/54 
In: Uhr, Wolfgang, Esswein, Werner \& Schoop, Eric (Hg.) 2003. Wirtschaftsinformatik 2003: Medien - Märkte - Mobilität, 2 Bde. Heidelberg: Physica-Verlag

ISBN: 3-7908-0111-9 (Band 1)

ISBN: 3-7908-0116-X (Band 2)

(C) Physica-Verlag Heidelberg 2003 


\title{
Etablierung von Wissensgemeinschaften in virtuellen Organisationen
}

\author{
Volkmar Pipek \\ Universität Siegen
}

\section{Markus Won}

Universität Bonn

Virtuelle Organisationen bilden durch die ihnen eigene Heterogenität und Autonomie der beteiligten Partner ein besonders anspruchsvolles Feld für technische Unterstützungsmöglichkeiten des Wissensmanagements. Wir leiten in diesem Beitrag das Konzept der "Knowledge Community" bzw. Wissensgemeinschaft sowohl aus theoretischen Überlegungen wie auch aus den Ergebnissen empirischer Untersuchungen in virtuellen Organisationen ab. Wir entwickeln Zielvorstellungen für technische Unterstützungskonzepte, diskutieren aber vor allen Dingen unsere Konzepte und Erfahrungen mit dem Prozess der Etablierung von Wissensgemeinschaften vor allen Dingen vor dem Hintergrund indiviueller Kosten-NutzenAbwägungen und vorhandener heterogener Infrastrukuren.

Schlüsselworte: Wissensmanagement, virtuelle Organisationen, Gemeinschaften

\section{Einleitung}

Virtuelle Organisationen (vgl. [Pi $\left.{ }^{+} 98\right]$, [Mo97]) bilden aufgrund der Flexibilität am Markt eine interessante Organisationsform, z.B. für den Dienstleistungsbereich (vgl. [Bul ${ }^{+}$97]). Gerade dort, wenn es z.B. um Beratungs-, Weiterbildungs- oder Softwareentwicklungsdienstleistungen geht, spielt das Wissen der beteiligten Akteure und der Umgang damit in der Durchführung der Kooperation eine entscheidende Rolle für den Gesamterfolg der Unternehmung. Und gerade diese Organisationsform stellt jedoch besondere Herausforderungen an das Wissensmanagement und seine technische Unterstützung.

Die Besonderheiten virtueller Organisationen gegenüber 'klassischen' Organisationsformen lassen sich wie folgt zusammenfassen (vgl. [ArHä95]):

- Autonomie: Es handelt sich um einen Zusammenschluss juristisch eigenständiger Personen und Organisationen. 
- Heterogenität: Die Partner unterscheiden sich nicht nur hinsichtlich ihrer Kernkompetenzen (was die Sinnhaftigkeit des Zusammenschlusses je erst begründet), sondern auch hinsichtlich ihrer Arbeits-, Kommunikations- und Kooperationsweisen, sowie ihrer technischen Infrastruktur und Vorbildung.

- Verteiltheit: Die Partner arbeiten räumlich verteilt, oft auch über größere Distanzen hinweg.

- Technische Vernetzung: Die Partner sind in irgendeiner Form durch Computernetzwerke verbunden und verlassen sich in der Kommunikation und Kooperation in starkem Maße auf neue Medien.

Diese Eigenschaften stellen, wie in der Literatur erwähnt (z.B. [Kreb98]) und durch eigene empirische Untersuchungen erhärtet, besondere Herausforderungen an Konzepte zur technischen Unterstützung des Wissensmanagements. Jedoch ist auch die Umsetzung von Veränderungsprozessen bzw. organisationaler Lernprozesse (vgl. [Seng01], [Orli00]), besonderen Herausforderungen unterworfen. In unserem Beitrag untersuchen wir die dort wirkenden Dynamiken und schlagen basierend auf unseren empirischen Untersuchungen das Konzept der "Knowledge Communities" bzw. "Wissensgemeinschaften" als sinnvolles sozio-technisches Instrument des Wissensmanagements in virtuellen Organisationen vor und skizzieren einen Etablierungsprozess für technische Plattformen. Abschließend beschreiben wir erste Erfahrungen, die wir in zwei virtuellen Organisationen sammeln konnten.

Wir wollen zunächst unsere eigenen Untersuchungen zum Wissensaustausch in virtuellen Organisationen beschreiben, und sie später mit Literatur in diesem Bereich abgleichen.

\section{Praxis des Wissensaustausches in virtuellen Organisationen}

Im Rahmen einer qualitativen Empirie in zwei virtuellen Organisationen haben wir 22 Einzelpersonen in unterschiedlichen Positionen und mit unterschiedlichen Aufgabenbereichen befragt. Die Organisationen arbeiten im Schnittfeld zwischen Beratungs- und Schulungsdienstleistungen und Softwareentwicklung. Die Organisationen wurden darüberhinaus über einen Zeitraum von zwei Jahren vor allen Dingen hinsichtlich der Verwendung neuer Technologien zum Informationsaustausch beobachtet. Dazu wurden sowohl weitere Einzelgespräche mit wichtigen Rollenträgern geführt, als auch insgesamt zwölf Meetings verfolgt, in denen die aktuellen Entwicklungen der Organisations- und Technikentwicklung diskutiert wurden. 
Als Befragungsmethode in den Interviews wurden offene, narrative Interviews zur Praxis ihrer branchenbedingt stark wissensorientierten Arbeit genutzt. Die einzelnen Interviews dauerten zwischen einer und drei Stunden, und waren in einen narrativen und einen semistrukturierten Teil unterteilt. Im ersten Teil ging es um eine Beschreibung der allgemeinen Arbeitssituation, im zweiten um Fragen zur Organisation und den verwendeten Technologien. Die beiden untersuchten Unternehmensberatungsnetzwerke (wir nennen sie hier SIGMA und GAMMA) verfügen über jeweils zwischen 150 und 300 Mitarbeiter und machen Umsätze im niedrigen zweistelligen Millionen-Euro-Bereich. Beide decken die Bereiche Training, Beratung und Softwareentwicklung ab, allerdings liegt bei SIGMA der Fokus auf Trainingsdienstleistungen, während GAMMA eher im Bereich Softwareentwicklung aktiv ist. Die untersuchten Organisationen decken unterschiedliche Teile des Spektrums virtueller Organisationen ab. SIGMA ist eine Netzwerkorganisation aus deutschlandweit verteilten Selbstständigen mit in der Regel Heimarbeitsplätzen. GAMMA ist in einer flachen Hierarchie organisiert und hat neben dem Hauptsitz noch fünf weitere Standorte im In- und Ausland, die z.T. autonom bestimmte Produkte weiterentwickeln und betreuen. Die Befragten (überwiegend männlich) waren von ihren Tätigkeiten her IT-Trainer, Projektleiter, Programmierer, Berater und Marketingmitarbeiter. Für uns waren die Kernfragen der Empirie darauf ausgerichtet, wie Informationen im Netzwerk weitergegeben werden bzw. welche Möglichkeit die Mitarbeiter haben, an für die aktuelle Arbeitsaufgabe relevantes Wissen zu gelangen. Dabei zeigte sich, dass fast in allen Fällen das persönliche Gespräch - sei es in der Teeküche oder auch am Telefon - an erster Stelle stand. Die Unternehmen bieten zusätzlich auch Informationsbasen (Dateisysteme, Datenbanken oder WWW-basierte Informationssysteme) an. Wir möchten hier anhand von drei Beispielen der Organisationspraxis Problemaspekte für die Nutzung neuer Medien zum Informationsaustausch näher beschreiben.

\subsection{Beispiel: Nutzung von Informationsspeichern}

Innerhalb der Organisation gibt es verschiedene Datenbanken, die im wesentlichen Projektdokumentation enthalten. Dabei handelt es sich z.B. um im Rahmen von Qualitätssicherungsmaßnahmen anfallende interne Reviews, sowie Projektabschlussberichte, Abrechnungsdaten und Angebotsdaten. In Teilgruppen fanden sich auch gemeinsame Adressbestände oder Termininformationen.

Die vorgehaltene Informationsbestände werden häufig nicht über das jeweilige Projekt hinaus genutzt. Sie werden meist mit niedrigem Aufwand verwaltet, nicht für eine Weiterverwendung aufbereitet (z.B. durch geeignete Indizierungen) und nicht weiter gepflegt. Die Befragten machten eine fehlende inhaltliche Zuständigkeit auch für eine schnelle Veraltung dieser Informationsquellen verantwortlich. Werden Informationen benötigt, so werden vorzugsweise Kollegen direkt angesprochen. Auf diese Weise lässt sich als Zusatzinformation auch eine Aussage über die Qualität der so gefundenen Materialien treffen. Auch transitive Beziehun- 
gen - also das Nachfragen über mehrere Stationen in Folge - führt zu den gewünschten Informationen. "Soziale Navigation" als Suchtechnik bei der Recherche ist ein beliebter und erfolgversprechender Ansatz der Informationsrecherche innerhalb der Netzwerke.

Auch für das Problem der Transparenz der in der virtuellen Organisation vorhandenen Kompetenzen wurden Profildatenbanken in beiden Organisationen in unterschiedlichen technischen Realisierungen ausprobiert. Auch diese Ansätze scheiterten alle am Problem der Informationswartung. Die Netzwerkpartner leiten entsprechende Informationen aus informellen Kommunikationen in den Organisationen ab (vgl. [Pipe+03a]).

Offensichtlich organisieren sich viele der Befragten aber eigene, individuelle Informationsspeicher, in denen sie gut navigieren können und häufig auch benötigte Informationen zu einem aktuellen Problem auffinden können. Dort kommt es zum Teil auch zu regelmässigen bilateralen Austauschprozessen, die sich meist entlang fest etablierter sozialer Beziehungen abspielen.

\subsection{Beispiel: Weitergabe von Schulungsmaterialien}

In SIGMA sind ein Großteil der Netzwerkpartner Einzelunternehmer im Bereich IT-Schulung. Der Umgang mit der Weitergabe selbsterstellter Materialien (z.B. Lehrmaterialien) wurde in den Interviews indirekt angesprochen. Auffallend war, dass es für alle Beteiligten kein Problem war, auf Anfrage Materialien innerhalb der Synergie zur Verfügung zu stellen, und das, obwohl Trainer durchaus Konkurrenten sein können. Wissenseignerschaft scheint so einerseits keinen hohen Stellenwert zu besitzen, andererseits verbinden viele Netzwerkmitglieder bestimmte Erwartungen mit der Weitergabe von Material:

- Rückflusskonvention: Wenn Materialien weitergegeben und verwertet wurden, sollte das Verwertungsergebnis an den Urheber der ursprünglichen Materialien zurückgehen.

- Feedbackkonvention: Wenn Materialien weitergegeben wurden, wird ein kompetenter Kommentar dazu erwartet.

- Kontextbereicherung: Mit der Weitergabe nur nach Anfragekontakt werden Informationen erwartet (z.B. neue Projekte/Kunden/ Entwicklungen), die zum einen einen Kontext über die Materialverwertung geben, zum anderen einen generellen Überblick über Entwicklungen des Netzwerkes im Ganzen vermitteln. Vereinzelt wurde auch der durch die Anfrage entstehende soziale Kontakt an sich positiv bewertet.

- Weitergabekontrolle: Auch wenn es innerhalb von SIGMA keine benannten Hemmnisse zur Materialweitergabe gibt, ist der Bereich der Weitergabe nicht beliebig. Eine harte Grenze ist die Unterscheidung nach SIGMA-interner und 
SIGMA-externer Weitergabe: Während interne Verbreitung unstrittig ist, ist externe Weitergabe meist eher geduldet als erwünscht. In Einzelfällen, in denen die Erstellung von Lehrmaterialien eigentlicher Gegenstand eines Auftrages war, wird dies explizit verurteilt.

Unklar ist, welche Erwartungen/Konventionen für Grauzonen bestehen, z.B. bzgl. der Weitergabe an Projektmitarbeiter, die nicht SIGMA-Mitglieder sind. Es wurde zunächst kein Bedarf für gruppenorientierte Repositories oder ähnliche zentrale Materialarchive gesehen. Jedes Mitglied von SIGMA betrachtet sich als „Portal“ seiner/ihrer Daten.

\subsection{Beipiel: Produktivarbeit vs. Infrastrukturarbeit}

Die Befragten in beiden Organisationen sehen sich naturgemäß auch einem organisationsinternen Wettbewerbsdruck ausgesetzt. Dabei wird auf indiviueller Ebene sehr genau abgewogen, wieviel Zeit man in "Infrastrukturarbeiten" investiert, d.h. in Arbeiten, die der Erhaltung der eigenen Wettbewerbsfähigkeit dienen, und wieviel Zeit in "Produktivarbeiten", die im Rahmen eines Kundenauftrages abrechenbar sind.

Mitglieder in SIGMA leben letztendlich von ihren Kontakten und ihrer persönlichen Kompetenz. Letztere weiterzuentwickeln (z.B. Projektleitung, Marktkompetenzen, Fachkompetenzen) wird auf unterschiedliche Weise erledigt. Marktkompetenz wird durch regelmäßiges Lesen von Zeitschriften bewältigt und fällt nur bei den Netzwerkmitgliedern ins Gewicht, die sich stark auf externe Auftragsakquisition eingestellt haben. Führungskompetenzen werden meist durch Seminare weiterentwickelt. Am häufigsten müssen Fachkompetenzen weiterentwickelt werden, was meist über Bücher oder existierende Schulungsmaterialien geschieht.

Die Mitglieder von SIGMA verlassen sich in stark unterschiedlichem Maße auf Technik. Grundausstattung ist meist der PC mit Anbindung an ein Intranet zum Nachrichtenaustausch. Soweit Laptops oder Palmtops (seltener) zum Einsatz kommen, müssen die unterschiedlichen Datenbestände, von Lehrmaterialien bis zu Kontakt- und Termindatenbanken synchronisiert werden. Zudem werden in unterschiedlichem Maße Sicherungskopien und Backups erstellt. Auch weitere Infrastrukturmaßnahmen wie z.B. Rechnerkonfiguration müssen durchgeführt werden. Auch die Weiterqualifikation hinsichtlich Techniknutzung gehört in diesen Bereich.

Wichtig ist auch die Beobachtung, dass auch Umorganisationsmaßnahmen des Netzwerkes und die Einführung neuer technischer Systeme durchaus kritisch gesehen wurde, da der damit verbundene Aufwand ebenfalls als individuelle Infrastrukturarbeit zu verbuchen war. Einzelne Netzwerkmitglieder entwickelten so auch sehr unterschiedliche Umgehensweisen mit dem Problem, von der völligen 
Ablehnung von Neuerungen bis zu sehr intensiven Auseinandersetzungen (vgl. [Tör $\left.\left.{ }^{+} 03\right]\right)$.

\subsection{Diskussion der Beobachtungen}

Wir müssen feststellen, dass die individuelle Kosten-Nutzen-Balance einen entscheidenden Einfluss auf die Akzeptanz von Neuerungen und somit für die Durchführung organisationaler Lernprozesse hatte. In SIGMA war z.B. das Scheitern des Versuchs der Etablierung einer gemeinsamen Groupwareplattform zu beobachten, die nur in Teilgruppen des Netzwerkes hinreichend Unterstützung fand (vgl. [Tör $\left.{ }^{+} 03\right]$ ). Wichtig ist dabei festzustellen, dass der hohe Grad an Autonomie der Beteiligten tatsächlich auch alle mit derartigen Prozessen verbundenen Konflikte ausbrechen lässt.

Generell müssen technische Lösungen für den Informationsaustausch offensichtlich sehr defensiv angelegt sein. Sie müssen sich eng an der aktuellen Arbeitspraxis und dem aktuellen Stand der Fähigkeiten der Techniknutzung orientieren. Es geht auch aus den Beobachtungen hervor, dass in der Abwägung von Kosten und Nutzen den Kosten ein höheres Gewicht beigemessen wird als den Nutzenfaktoren (Originalzitat: "Was soll mir das bringen? Meine Arbeit bekomme ich so bisher auch gut erledigt."). Unterschätzt wurde auch, dass die Etablierung einer gemeinsamen Arbeitspraxis (mediiert durch die gemeinsame Nutzung eines neuen Tools) durch die starke Heterogenität im Netzwerk größere Anpassungsanstrengungen verursacht als in klassischen Organisationsformen.

Die Wartung von Informationen wird offensichtlich ebenfalls als Overheadarbeit empfunden, die man ohne konkreten Anlass nicht erledigt (Beispiel Kompetenzdatenbanken, vgl. auch [Pipe $\left.{ }^{+} 03 \mathrm{a}\right]$ ]). Auch eine für die Wiederverwendung adäquate Aufbereitung von Informationen fand häufig nicht statt. Die Dominanz sozialer Faktoren im Informationsaustausch ist ebenfalls ein wichtiges Merkmal von Informationsaustauschprozessen. Vertrauen in die Einhaltung von Konventionen war bei SIGMA ein wichtiger Punkt für die Weitergabe von Materialien. Dem Anfragekontakt selber wurde eine große Bedeutung für die Verbreitung informeller Informationen beigemessen. Dies lässt einen Ansatz der Etablierung zentraler "Wissensarchive" nicht besonders attraktiv erscheinen.

Auf Basis dieser Ergebnisse liegt der Schluss nahe, Wissensmanagement für virtuelle Organisationen vorrangig nicht durch Strategien zur Informationsspeicherung und zum Information Retrieval zu unterstützen, sondern der Unterstützung wissensbezogener Kommunikationen ein größeres Gewicht zu geben. Wir haben diesem Aspekt und der Wichtigkeit sozialer Aspekte durch das Aufgreifen des Community-(Gemeinschafts-)Begriffs Rechnung getragen, an dem sich unser Konzept orientiert. 


\section{Technische Unterstützung des Wissensmanagements}

Der Begriff des Wissens ist in der Literatur und vor allem in der Praxis sehr unterschiedlich besetzt. Willke ([Will99]) unterscheidet zwischen Daten als kodierte Beobachtungen, die in einem systemischen Kontext zu relevanten Informationen werden. Wissen kann dann als im persönlichen Erfahrungsschatz verankerte Information gesehen werden. Wie auch Willke wollen wir im folgenden Wissen (oder auch Expertise, Erfahrung, Kompetenz) als aktiv verarbeitete und durch persönliche Erfahrung gestützte Information verstehen. Informationen sind somit die Bausteine oder Elemente des Wissens, jedoch erst ein systematisches Management und eine geeignete Verarbeitung dieser Elemente ermöglichen individuelles Lernen und erfahrungsgestütztes Wissen.

Wissensmanagement wurde durch von Krogh und Venzin ([vKVe95]) durch verschiedene Aufgaben charakterisiert: Sammeln vorhandenen Wissens, Verfügbarmachen von Wissen, Erleichtern der Wissensgenese, Verbreitung von Metawissen über Organisationswissen und Operationalisierung von Wissen für Produkte und Dienstleistungen. Die technische Unterstützung des Wissensmanagements von Organisationen orientierte sich meist an der Metapher des „Organisationsgedächtnisses“ ([WaUn91], [Fais96]) und bildete große, häufig vernetzte Informationsräume heraus (z.B. [ $\left.\mathrm{Aks}^{+} 98\right]$ ). Dabei sind durchdachte Ablagestrukturen ebenso wichtig, wie mächtige Navigations- oder Suchfunktionen. Ergänzend zu Datenbank-orientierten Ansätzen stehen Ansätze des Information Retrieval ([BYRN99]), das damit befasst ist, große Datenbestände mit intelligenten Suchverfahren handhabbar zu machen.

Diese eher datenspeicherungsorientierten Ansätze wurde aus verschiedenen Richtungen kritisiert. Bannon und Kuuti ([BaKu96]) werteten diese Lösungen als zu statisch, zu passiv und zu isoliert von der Praxis wissensintensiver Arbeitsprozesse. Andere maßen der Kommunikationsunterstützung einen größeren Stellenwert im Wissensmanagement $\mathrm{zu}$ als der reinen Vorhaltung von Informationen ([AcMc96] [Shum97], [SuSh98], Übersicht in [PiWo02]), wiesen auf die Grenzen explizierbaren Wissens hin ([NoTa95]) und auf die Notwendigkeit der Unterstützung der kollaborativen Veränderung virtueller Informationsräume ([Shum97]). In vielen der entwickelten innovativen Konzepte geht es darum, neben Informationen auch Experten sichtbar und auffindbar zu machen (z.B. [AcMc96]). Daneben sind immer auch Vertrauens- (z.B. [Hand95]) und organisationspolitische Aspekte (z.B. [Pipe $\left.{ }^{+} 03 \mathrm{~b}\right]$ ) wichtige Einflussfaktoren. 


\section{Knowledge Communities in Virtuellen Organisationen}

Der Community-Begriff (mit "Gemeinschaft, Gruppe" u. E. nur unzureichend übersetzbar) ist in den wissenschaftlichen Diskussionen vielfältig besetzt (z.B. [Weng98], [Les $\left.\left.{ }^{+} 00\right]\right)$. Wir verstehen unter einer Community eine nicht notwendigerweise organisierte Gruppe von Akteuren, die ein gemeinsames Interesse oder eine gemeinsame Einstellung teilen, sich dieses Interesses bzw. der Einstellung bewusst sind und über dieses Interesse bzw. diese Einstellung mit anderen prinzipiell interagieren wollen.

Im Zusammenhang mit Organisationen assoziieren wir mit dem Begriff der Community informale Gruppenstrukturen, die sich in Organisationen über Abteilungsgrenzen und Rollenverhältnisse hinweg entwickeln, um zu einer aufgabengerechten Arbeitspraxis zu kommen. In unseren empirischen Arbeiten ergaben sich Beispiele, in denen über solche Communities z.B. arbeitskontextrelevante Informationen transportiert wurden, wie Urlaubszeiten, Neukunden, etc. Frühere Arbeiten haben allerdings bereits herausgestellt, welche wichtige Bedeutung diese informalen Strukturen für die Verbreitung von Wissen und Handlungskompetenzen haben können. Orr ([Orr90]) beschrieb die korrektive Wirkung einer Community von Servicetechnikern eines Kopiererherstellers, durch die den Fehlinterpretationen der Servicehandbücher und der dort skizzierten Entscheidungsbäume für die Gerätediagnose entgegengewirkt wurde. Die relevanten Kriterien der Praxis dieser Community beschrieb Orr mit dem Trias Erzählungen ("Narrations", in der Community verbreitete Geschichten über Fehlversuche und Missverständnisse), Zusammenarbeit ("Collaboration", als die dominierende Metapher der Arbeitsaktivitäten. Selbst wenn die Arbeit vor Ort von einem Servicetechniker allein verrichtet wurde, war der damit verbundene Problemlöseprozess von kollaborativer Natur.) und Soziale Konstruktion ("Social Construction", die Entwicklung gemeinsamer Sichtweisen und Verständnisse über wichtige Aspekte des Aufgabenkontextes, z.B. Handbücher und Kopierer). Die technischen Unterstützungsmethoden für das Wissensmanagement sollten Aktivitäten im Rahmen dieses Trias unterstützen.

Brown und Duguid ([BrDu00]) leiten sowohl für das Wissensmanagement als auch für das Organisationale Lernen im Allgemeinen das Leitbild der Organisation als "Community-Of-Communities" ab, dem wir uns mit unseren Arbeiten anschließen.

\subsection{Zielvorstellung Knowledge Community}

Unser Begriff der „Knowledge Community“ meint einen Zusammenschluss von Mitgliedern/Einheiten von VO (und damit eine Perspektive auf VO), der sich zunächst nicht an Marktgegebenheiten, Organisationsstrukturen oder regionaler Standortverteilung orientiert, sondern ausschließlich an der Expertise (Wissen, 
Kompetenzen oder Interessensgebiete) von beteiligten Mitgliedern und Einheiten. Knowledge Communities sind demnach geeignet, eine Gruppe entsprechend gemeinsamer Interessen zusammenzuführen und den Wissensaustausch untereinander anzuregen.

Verschiedene Studien zum Wissensmanagement weisen vor allen Dingen auf die Situiertheit von Wissen und Lernen hin (vgl. Kurzübersicht in [Weng98], S. 13), und darauf, das Umgebungen entsprechend ausgestaltet werden ([AcHa98], [Lees97]). Das Leitbild des „Organizational Memory“ greift auch hier zu kurz, da es zu abstrakt ist, um konkrete Hinweise auf die Ausgestaltung technischer Unterstützungssysteme für Knowledge Communities zu liefern. Wir orientieren uns an der Leitmetapher der Wissenslandschaft, in der die Akteure bezogen auf Situation und Perspektive ein ihrer aktuellen Arbeitsaufgabe oder Problemstellung angemessenes Panorama vorfinden. Obwohl dieses naturgemäß nicht nur Artefakte neuer Technologien, sondern auch z.B. Aufzeichnungen, Telefonlisten, Handbücher, etc. im Arbeitsarrangement enthält, sollten technische Unterstützungssysteme so konstruiert sein, dass bezüglich eines „Online-Arbeitsarrangements“ einer Gruppe von gleich Interessierten die größtmöglichen Gestaltungsfreiheiten eingeräumt werden. Im Rahmen des Selbstorganisationsprozesses einer solche „Knowledge Community“ soll sich dann ein angemessenes Arrangement entwickeln können. Ein entsprechendes Unterstützungssystem sollte dieöffentliche Wissenskommunikation unterstützen, Wahrnehmungs- und Präsentationsmöglichkeiten für vorhandenes Wissen und Expertise eröffnen, Wissensarbeit aufgaben- und gruppenangemessen verfolgbar machen und die autonome Administration von Gruppen durch endbenutzergerechte Anpassbarkeit unterstützen.

Nur mit Hilfe eines derartig flexiblen Unterstützungssystems als Zieltechnologie der Etablierung von Knowledge Communities kann man dem Aspekt der Situiertheit wissensintensiver Arbeiten gerecht werden.

\subsection{Fehlende Etablierungskonzepte}

Knowledge Communities können also als eine Art von Gruppe betrachtet werden, die sich durch ähnliche Interessen definiert und innerhalb welcher Wissen offen ausgetauscht wird. Dies ist grundsätzlich ein Leitbild, das von den meisten Wissensmanagement-Ansätzen mitgetragen wird (s.o.). Offen bleibt jedoch die Frage, wie sich eine Knowledge Community systematisch unterstützt etablieren lässt. Es orientiert sich an der Entstehung von Gruppen im Allgemeinen, versucht jedoch durch Interventionen und äußere Steuerung die oben beschriebenen Schwierigkeiten zu umfahren. 


\section{Prozess der Etablierung von Knowledge Communities}

Das Ziel des hier beschriebenen Vorgehens ist es, eine Sichtbarkeits- und Interaktionssphäre zu schaffen, die sowohl eine Transparenz über vorhandene Expertise schafft als auch Anlaufstelle für konkrete Nachfragen sein kann, und so das organisationale Lernen fördert. Zur Etablierung solcher Strukturen haben wir ein Konzept erarbeitet und für erste Erfahrungen auch umgesetzt, das wir im Folgenden beschreiben wollen. Abb. 1 stellt das vierphasige Vorgehensmodell schematisch dar. In den hier vorgestellten Knowledge Communities soll in der Gruppe praxisrelevantes Wissen ausgetauscht werden. Dies kann innerhalb einer Organisation geschehen. Häufig finden sich Experten aber auch über einen längeren Zeitraum in organisationsunabhängigen Austauschforen wieder.

Für uns ist dabei wichtig, dass die eingesetzte Technik einfach zu bedienen ist und auf Standards aufsetzt. Ersteres erleichtert den Zugang für alle Beteiligten und erlaubt es, dass sich die Beteiligten in erster Linie auf inhaltliche Probleme konzentrieren können und nicht so sehr mit den technischen zu kämpfen haben; Zweiteres

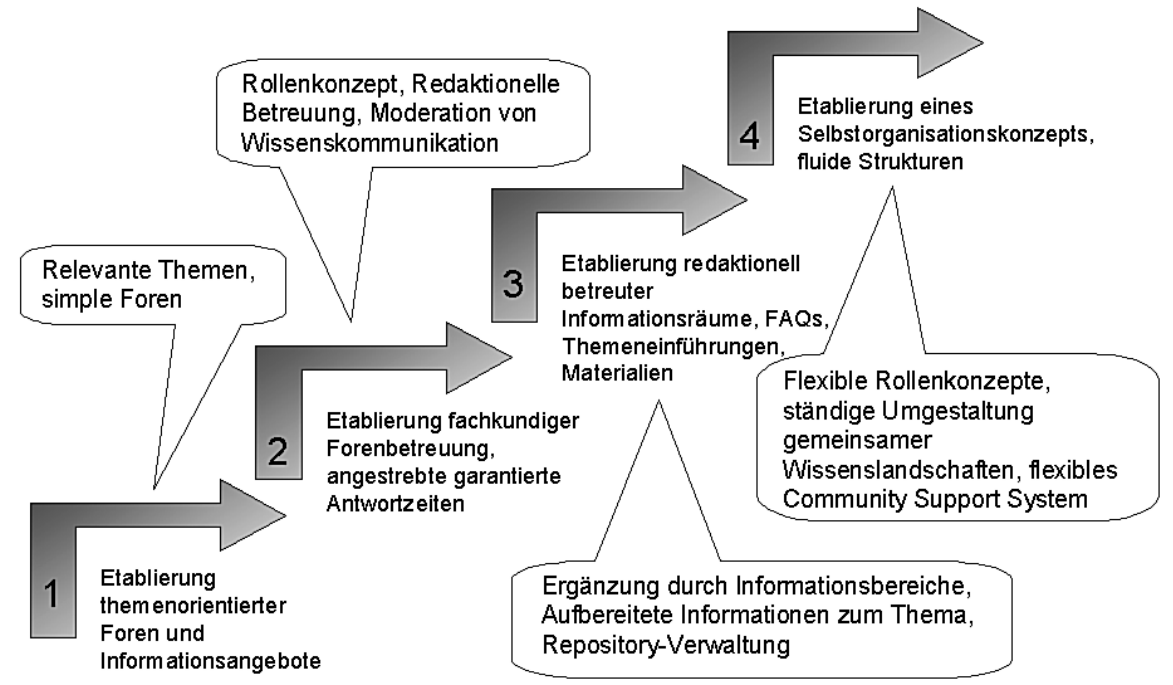

Abbildung 1: 4-Phasen-Modell zur Etablierung von Knowledge Communities

ist vor allem im Kontext der virtuellen Organisationen wichtig. Die technische Basis muss für alle zugänglich und bestenfalls auch ohne hohen Installations- und Wartungsaufwand einsetzbar sein. Gerade wenn sich solche Gemeinschaften über Organisationsgrenzen hinweg etablieren sollen, spielt dies eine wichtige Rolle. Es ist für die Umsetzung auch nicht nötig, eine eigene Plattform aufzusetzen. Zu Beginn kann mit einfachen Techniken wie Mailinglisten oder Internet-basierten Fo- 
ren gearbeitet werden. Unterschiedliche Techniken können später auch parallel zueinander eingesetzt werden. Weiterhin setzen die vier Phasen so aufeinander auf, dass möglichst zu jedem Zeitpunkt und für alle Beteiligten ein positives Kosten-Nutzen-Verhältnis gilt, indem in aufeinander aufbauenden Phasen der Arbeitseinsatz in das Wissensmanagementsystem und dessen Nutzen sukzessive gesteigert werden soll. So entgeht man dem Problem, in der Initiierungsphase zu viele potentielle Nutzer zu verschrecken. Gerade in virtuellen Organisationen, in denen der eigene Aufwand sehr kritisch beobachtet wird, kann dies helfen, ein dauerhaftes und stabiles System für den Wissensaustausch zu etablieren.

Nicht immer müssen alle Phasen durchlaufen werden, um eine Gruppe zu einer funktionierenden Knowledge Community zusammenwachsen zu lassen. Die "Abarbeitung" der einzelnen Phasen richtet sich vielmehr nach Art der diskutierten Themen, Enge der Zusammenarbeit und auch der Größe und Gestalt der Gruppe. So lassen sich beispielsweise Gruppen mit häufig wechselnden Mitgliedern kaum durch ein hochspezialisiertes, technisches System unterstützen, da der Einarbeitungsaufwand dann dem Nutzen übermächtig gegenüberstünde. Wie weit also dieses Etablierungskonzept verfolgt werden soll, muss in der Gruppe diskutiert und entschieden werden.

\subsection{Schritt 1: Etablierung von Themenforen}

In Schritt 1 werden themenorientierte Foren eingeführt. Sie dienen dazu, relevante Themen innerhalb der Gruppe aufzuspüren und sollen den Zugang zu textbasierter Kommunikation in offenen Diskussionsräumen erleichtern. Außerdem kann externer Input (z.B. aus Newstickern oder Mailinglisten) zusätzlich eingespeist werden. Auf technischer Seite sind die Anforderungen gering. Basisfunktionen, wie das Definieren eines Themas, das Senden einer Nachricht, die Teilnahmeerklärung zu interessanten Themen und natürlich das Lesen des Forums stehen zur Verfügung. Die technische Realisierung sollte hier hinreichend flexibel sein, um ein an die Anforderungen angepasstes Design zu ermöglichen. Hier spielt auch das Design des Front-Ends und die Gestaltung der Interaktionspunkte eine wichtige Rolle. Sie sind grundlegend wichtig für die Akzeptanz des Systems.

Vorteil bei diesem Vorgehen in Stufe 1, in der die Teilnehmer noch keine oder nur einige Erfahrungen mit dem IuK-gestützten Wissensaustausch haben, ist die nur schwache Außensteuerung (vor allem auf technisch-administrativer Seite), die alle Beteiligten zu Wort kommen lässt und eigene Gestaltungsmöglichkeiten erlaubt. Themen, Unterthemen, die Art der Beiträge und auch die Führung einer Dokumentation werden durch die Teilnehmer gesteuert. Die vorausgesetzte technische Flexibilität ermöglicht es, die von den Teilnehmern geforderte Funktionalität schnell umzusetzen. Auch auf organisatorischer Ebene - die Teilnehmer bestimmen gemeinsam die Themen - wirkt dieser flexible Ansatz, indem es durch stän- 
dige Rückkopplungen "bedarfsgerecht" Themen in den Vordergrund rückt oder andere zurückbleiben.

Das skizzierte Vorgehen erfordert jedoch viel Zeit und Mühe und aus Sicht der Teilnehmer Kosten. Durch das geringe Maß an Steuerung ist ein längerer Einschwingvorgang die Regel. Dieser Aufwand - der selbst eine Qualität in sich birgt - könnte verringert werden, indem die Themen nicht mehr im Forum selbst ausgehandelt werden, sondern vorher durch Befragungen aller Beteiligten ermittelt werden. Hierdurch sinkt die Selbststeuerung vor allem in der Anfangsphase, die naturgemäß durch Unsicherheit gegenüber den anderen Teilnehmern, der eingesetzten Technik und dem Prozess selbst gekennzeichnet ist. Problematisch können sich hier durch schlecht gewählte Befragungstechniken oder eine zu geringe Befragungsintensität nicht-repräsentative Themenwünsche ergeben, die dann wiederum zu einer geringen Beteiligung führen.

Gegen Ende der ersten Phase sollte damit begonnen werden, die Ergebnisse zu sammeln, nachhaltig aufzubereiten und durch externe Informationsquellen anzureichern. Ein gruppeninterner News-Letter kann hier eine Strukturierungshilfe darstellen. Aus dieser Beschreibung lassen sich dann auch die Kriterien, anhand derer sich der Erfolg des Konzepts "messen" lässt, beschreiben.

- Themenvisualisierung: Wurden relevante Themen herausgearbeitet und voneinander abgegrenzt und im weiteren Verlauf weiter ausdifferenziert?

- Signifikanter Bekanntheitsgrad: Ist das Forum allen Beteiligten bekannt und auch die Nutzung geläufig?

- Beteiligung: Lässt sich anhand der Einträge im Forum ausmachen, dass die gesamte Gruppe an den Diskussionen beteiligt war? Zeitlicher Verlauf?

\subsection{Schritt 2: Etablierung redaktioneller Betreuung}

Phase 2 des Vorgehensmodells ist durch eine Qualitätsverbesserung der Informationen und Akzeptanzsteigerung des Mediums charakterisiert. Dies sollte durch die Einführung redaktioneller Betreuung erreicht werden, die sich in Form von inhaltlicher Selektion, pointierte Darstellung von Wichtigem und auch Kommentaren zeigt. Die damit verbundene redaktionelle Arbeit sollte innerhalb der Gruppe gesteuert und erledigt werden. Abstimmungsprozesse, wer diese Aufgaben übernehmen kann und wird, sollten über das System geführt werden. Die Findung redaktionell Verantwortlicher zur Forenbetreuung geschieht dementsprechend nach den Prinzipien Eignung, Akzeptanz, Freiwilligkeit und Präsenz im Medium. Sie kann als erster Schritt zur Bildung eines Rollenmodells innerhalb der Gruppe betrachtet werden. Diese fluiden Rollen ermöglichen dann auch eine Unterscheidung nach Experten, Administration etc. Diese Rollenmodelle sollten technisch unterstützt werden in der Form, dass sie im Forum mit geeigneten Visualisierungskonzepten untermauert werden. Hier zeigt sich, wie wichtig es ist, schon zu Beginn 
ein mächtiges, aber flexibles technisches System auszuwählen, so dass solche Erweiterungen leicht umsetzbar sind.

Voraussetzung für die Einleitung der zweiten Phase ist vor allem eine gewisse Grundakzeptanz des forenbasierten Austauschs, sowohl was die technische Plattform angeht als auch die Fähigkeit zur Selbstorganisation und Bereitschaft zur öffentlichen Diskussion.

Durch die redaktionelle Betreuung wird die Verbindlichkeit der Diskussion erhöht und die Qualität der eingebrachten und evtl. überarbeiteten oder kommentierten Beiträge steigt. Weiterhin kann die Redaktion über garantierte Antwortzeiten dazu beitragen, die Bedeutung des Forums als Informationsquelle aufzuwerten. Auf der anderen Seite muss behutsam mit einer zu starken Steuerung durch die Redaktion umgegangen werden. Sie kann im Rückschluss auch wieder die Beteiligung absenken, da alle Arbeiten an „die Verantwortlichen“ delegiert werden. Wichtig ist hier, ein gesundes Mittelmaß zu finden und die Redaktion auch auf ihre Rolle als Motivation zur Teilnahme hinzuweisen.

Wie schon oben angesprochen, sind die Ziele der zweiten Phase, vor allem die Akzeptanz der neu geschaffenen Wissensaustauschplattform weiter zu fördern und gleichzeitig sowohl die Qualität als auch die Quantität der Beteiligung anzuheben. Die zweite Phase kann demnach als erfolgreich umgesetzt angesehen werden, wenn die Foren bei allen Beteiligten in die Arbeitspraxis integriert sind. Weiterhin ist auch wichtig zu beobachten, inwieweit es eine Gruppenidentifikation gibt. Dies ist besonders wichtig für den intensiveren Austausch, da es die Grundlage für ein gemeinsames Bild und damit einhergehend die Basis für einen vertrauten Umgang miteinander darstellt.

\subsection{Schritt 3: Ergänzung einer Wissenslandschaft}

Im weiteren Verlauf des Etablierungsprozesses ist das Ziel, eine Qualitätssteigerung der zur Verfügung stehenden Informationen durch eine Erweiterung der Informationsraumes zu erreichen. Während die ersten beiden Schritte stark auf der Kommunikation als Medium des Wissensaustauschs setzen, geht es nun darum, die vormalig ausschließlich zur Diskussion eingesetzte Infrastruktur nun auch als Dokumentenaustauschbasis einzusetzen. Gleichzeitig sollte eine weitere Verfeinerung der Interaktionsräume einsetzen. Dies geht üblicherweise mit der Menge der im Forum enthaltenen Beiträge einher. Sie fordert neue, verbesserte und auch feinere Themenstrukturen. Die bereitgestellten Materialien können von Mitgliedern der Gruppe selbst erzeugt worden sein, aber auch externe Materialien wie Verweise auf nützliche Web-Seiten sind hier denkbar. Wichtig ist, dass diese sich möglichst nahtlos in die durch die Kommunikationsstränge geprägten Strukturen integrieren lassen. Durch diese Erweiterung ergeben sich neue Anforderungen sowohl auf organisatorischer als auch auf technischer Seite und bei allen Beteiligten. 
Technisch sollte die eingesetzte Software den Upload von Dokumenten zulassen. Dies sollte innerhalb der Kommunikationsräume möglich sein. Optimalerweise ist die Einrichtung gemeinsamer virtueller Arbeitsbereiche erlaubt, wie es von diversen Groupware-Systemen (z. B. [Klöc02]) bekannt ist. Die bisher gewachsene Vertrauensbasis kann nun genutzt werden, um auch (gemeinsam) erarbeitete Materialien auszutauschen und allen Beteiligten auf der Plattform zur Verfügung zu stellen. Diese Veränderung der Arbeitsweise ist umso schwieriger, wenn die Gruppe sich aus Mitgliedern unterschiedlicher Organisationen zusammensetzt. Hier spielen dann auch organisationsinterne Verhaltensregeln und Privatheitsaspekte eine wichtige Rolle. Mit dem wachsenden Informationsangebot muss eine Professionalisierung der Foren-/Bereichsbetreuung einhergehen. Nicht nur der Aufwand, sondern auch das benötigte thematische Wissen steigt an, um die Qualität weiterhin sicherzustellen und den gewachsenen Ansprüchen aller Beteiligten gerecht zu werden.

Aus dem hier skizzierten Vorgehen wird deutlich, dass sich nun eine weitere Erhöhung des Nutzwertes über spezifische Angebote (z.B. für Neueinsteiger) und Transparenz (FAQ - Regelmäßig gestellte Fragen, Materialien) ergibt. Zusätzlich eingebrachte Materialien und der Gedanke, innerhalb einer vertrauten Gruppe Materialaustausch und -wiederverwendung betreiben zu können, eröffnet neue und bessere Nutzpotentiale.

Problematisch wirken sich vor allem die steigenden zeitlichen oder auch finanziellen Kosten aus, die mit steigender Professionalisierung und Mehrarbeit einhergehen. Die Einführung und Pflege der gemeinsamen Arbeits- und Ablagebereiche bringen neue Aufgaben mit sich und erhöhen den Orientierungsaufwand für alle Beteiligten. Vor allem die Redaktion oder Administration hat nun neue Aufgaben, wie die Wahrung der Übersichtlichkeit, die Sichtung und Pflege der Dokumente (neue Materialien sollten geprüft werden, FAQ, Einsteigerdokumentation müssen regelmäßig aktualisiert werden) und die Aushandlung, unterschiedliche Agreements bzgl. Materialverwendung zu übernehmen.

Am Ende der dritten Phase ist eine interaktive Informationsinfrastruktur unter starker Einbindung der Beteiligten entstanden. Hier werden wechselnde Themen zwischen den Beteiligten diskutiert, Materialien zur Verfügung gestellt und im Besonderen auch gemeinsam erstellt. Messgrößen für die Güte des Informationsraums können die Menge, die empfundene Qualität und Aktualität der bereitgestellten Informationen sein.

\subsection{Schritt 4: Selbstorganisierte Knowledge Community}

Die letzte Phase hat nun zum Ziel, das Maß an Selbststeuerung weiter zu erhöhen und die herausragende Bedeutung der Rollen „Administration“ und „Redaktion“ bzw. „Moderation“ abzuschwächen. Die Gruppe sollte mit technischer Hilfe zu einer selbsttragenden Plattform finden. 
Damit geht entsprechend auch eine weitere Verfeinerung der technischen Plattform einher. Vorhandene Interaktionsräume werden um zusätzliche technische Möglichkeiten zur flexiblen Selbstorganisation der IuK-Plattform selbst ebenso wie die technische Unterstützung bei der Organisation der Gruppe erweitert. Dementsprechend wird der Automatisierungsanteil weiter steigen, um die Gruppe von solchen Arbeiten zu entlasten. Auch völlig neue Funktionalitäten zur Selbstmoderation der Gruppenarbeit werden integriert. Dies reicht von einfach zu bedienenden Rollen/Zugriffsrechtkonzepten und entsprechendem Frontend bis hin zur ,automatischen“ Benennung/Wahl von Expertengremien, die den Moderationsaufwand verringern.

Voraussetzung für eine erfolgreiche Umsetzung dieser vierten und letzten Stufe ist vor allem eine hohe Akzeptanz des Mediums Computer und Kompetenz im Umgang damit bei allen Beteiligten. Aber auch der Gruppenkultur werden besondere Anforderungen in Bezug auf das Vertrauen untereinander abverlangt. Ein derartig offener Umgang miteinander, der es - wie schon in der zweiten und dritten Phase immer wieder gefordert werden - ermöglicht, Kompetenzen ebenso wie Inkompetenzen offen zulegen, muss es jetzt auch noch ertragen, Spezialisierungen zu tolerieren.

Gelingt dies, so ist das Ergebnis eine selbstorganisierte Gruppe, die sich schnell und flexibel neuen Marktgegebenheiten anpassen kann. Weitgehend alle Teilnehmer sind in den gemeinsamen Wissensaustausch integriert. Auch die Integration der individuellen Arbeit (Wissensgenese) in die Gruppe ist leicht möglich und schafft (vor allem auch durch das Offenlegen von Kompetenzdefiziten) neue Möglichkeiten der Zusammenarbeit. Problematisch ist vor allem, dass es nur schwierig möglich ist, neue Mitglieder in diese Gemeinschaft zu integrieren. Sie haben nicht den beschriebenen Erfahrungshintergrund. Auch auf Seiten der Gruppe, die sich über einen langen Zeitraum zusammengefunden hat, kann eine neue Person als Fremdkörper wirken und so den Zusammenhalt des Systems und die offene Kultur in ihm gefährden.

Phase vier endet mit der Etablierung einer Knowledge Community, in der das Verhältnis zwischen dem Eigenaufwand der Beteiligten und dem organisierten bzw. professionalisierten Gruppenaufwand zwischen den Mitgliedern der Gruppe ausgehandelt ist und wird. Sie ist hochflexibel und kann durch ein hohes Maß an Selbststeuerung schnell und erfolgreich Anpassungen entsprechend neuer Marktgegebenheiten umsetzen.

\subsection{Diskussion}

Das dargestellte Konzept versucht beginnend mit einem kommunikationsbasierten Ansatz ein Wissensmanagement-System in einer Gruppe zu etablieren. Dabei setzen vier Phasen, in denen sowohl der Aufwand und der Nutzen für alle Beteiligten steigt als auch die eingesetzte Technik komplexer und zugleich mächtiger wird. Es 
lässt sich also verallgemeinernd sagen, dass die Beteiligung der Gruppe zunimmt. In gleichem Maße sollten Umfang und Qualität der eingebrachten Informationen steigen. Das Modell stellt dafür die erforderlichen Mechanismen zur Verfügung. Auf der anderen Seite wächst auch die eingesetzte IuK-Technik mit den Ansprüchen. Während hier zu Beginn lediglich Mailinglisten oder andere vergleichsweise einfache Techniken zum Einsatz kommen, wird in der letzten Stufe durch Groupware-Systeme eine enge Zusammenarbeit ermöglicht.

Das Konzept verfolgt im Verhältnis zu klassischen Wissensmanagement-Ansätzen einen anderen Weg, was die technische Unterstützung eines Vertrauensmanagements angeht. Während dort Vertrauen zwischen Mitgliedern der Gruppe primär über Zugriffsrechte modelliert wird, steht bei diesem Ansatz die soziale Interaktion über Kommunikation im Vordergrund. Hier wird also ein vertrauensvoller Umgang nicht technisch gesichert, sondern der Aufbau eines Vertrauensverhältnisses der Beteiligten untereinander unterstützt. Dies hat den Vorteil, dass Zugriffsrechtsysteme immer recht starr wirken, der hier verfolgte Ansatz hingegen flexibel. Weiterhin ist ein persönliches Vertrauensverhältnis auch bedingt transitiv, wie sich in unseren Erhebungen im Rahmen des Projekts gezeigt hat. Solche Vertrauensverhältnisse geeignet zu visualisieren, kann dann Aufgabe einer technischen Plattform sein.

\section{Erste Erfahrungen}

Das hier vorgestellte Modell zur Etablierung von Knowledge Communities wurde entwickelt basierend auf den Beobachtungen in den Anwendungsorganisationen unseres Projektes. Die beschriebenen Maßnahmen wurden dabei durchgeführtnoch nicht in ein ganzheitliches Konzept eingebettet -, um die Etablierung zu forcieren. Problematisch bei der Betrachtung ist die auf drei Jahre begrenzte Laufzeit des Projekts, so dass bisher nicht der gesamte Prozess durchlaufen wurde. Stattdessen sind zwei Anwendungsfelder mit der Etablierung ihrer organisationsinternen Knowledge Communities derzeit mit der Umsetzung der zweiten Phase befasst. Die in Phase 3 und 4 diskutieren Schritte wurden jedoch mit Mitgliedern der Anwendungsorganisationen diskutiert. Dennoch können und sollen hier schon erste Ergebnisse präsentiert werden, wobei der Fokus darauf liegt, Probleme bei der Umsetzung zu schildern.

Das Finden eines für alle Beteiligten gleichermaßen nutzbaren IuK-Systems scheint zu Beginn eines der technischen Hauptprobleme darzustellen. Die meisten Organisationen verfügen über eine schon eingesetzte Infrastruktur. Die Eignungsprüfung dieser Plattform für die bevorstehenden Aufgaben wird hier meist durch die organisationsinterne IT-Abteilung vorgenommen. Hier spielen verschiedene, teils auch politische Probleme in die Entscheidungsfindung mit hinein. Zusätzlich wird dies dann nochmals erschwert, wenn versucht wird, eine Knowledge Com- 
munity über Unternehmensgrenzen hinweg zu etablieren. Hier zeigt sich die Inkompatibilität verschiedener Systeme zueinander. Auch der Wechsel zwischen mehreren Systemen - einige Benutzer arbeiten in unterschiedlichen Kontexten und in mehreren orthogonal zueinander stehenden Knowledge Communities - erschwert die Integration der Community-Arbeit in die tägliche Arbeitspraxis. Neben der Offenlegung von Kompetenzen, was auch technisch gestützt auf Basis von abgelegten Kompetenzprofilen oder mit Hilfe eines Expertise Awareness Systems (vgl. [Pipe $\left.{ }^{+} 03 a\right]$ ) unterstützt werden kann, kann auch die Offenlegung von aufzuarbeitenden Inkompetenzen ein wertvolles Wissen für die Gruppe sein. Dies kann beispielsweise in gezielten Weiterqualifikationsbemühungen münden. Allerdings hat sich in unseren Anwendungsfeldern gezeigt, dass zwischen der Darstellung von Kompetenz und der von Inkompetenz ein weiter Graben liegt. Hier spielen Ängste um die Positionierung im internen Markt eine Rolle.

Die in Phase zwei geplante redaktionelle Betreuung wurde in den Anwendungsfeldern eingeführt. Hier klafft jedoch, vor allem was die Antwortzeiten angeht, eine große Lücke zwischen denjenigen, die diese Arbeit leisten und denjenigen, die zu einem Thema qualifiziert Auskunft geben können. Während kleine Gruppen durch ihre Übersichtlichkeit diesem Problem noch Herr werden, gilt dies für größere Gruppen nicht unbedingt. Hier hilft zwar eine Professionalisierung der Redaktion insofern, als dass Antwortzeiten generell garantiert werden können. Jedoch zeigt sich häufig, dass die wirklich benötigten Informationen nur von Experten geliefert werden können, die aber auch durch die Einbindung in die Projektarbeit und die damit verbundene hohe Arbeitsauslastung solche Zeiten nicht einhalten können. Denkt man zudem an organisationsübergreifende Gemeinschaften, stellt sich die Frage, wer die redaktionelle Betreuung angesichts der fehlenden Möglichkeit des finanziellen Ausgleichs oder der zeitlichen Freistellung leisten kann oder möchte. Hier ist Verständnis der jeweiligen Organisationsleitungen notwendig. Weiterhin spielt hier auch die Angst eine Rolle, anderen zuviel des organisationalen Wissens preiszugeben.

Insgesamt zeigt sich, dass das geplante Vorgehen erfolgsversprechend scheint. Es liegt jedoch auch am weiteren Vorankommen des Etablierungsmodells, inwieweit verlässliche Aussagen für diese Einzelfälle gemacht werden können. Zudem muss im Folgenden die Empirie diesbezüglich auf eine breitere Basis gestellt werden.

\section{Zusammenfassung und Ausblick}

Dieser Beitrag beschreibt einen Ansatz, wie sich klassische WissensmanagementKonzepte mit dem Ansatz eines kommunikationszentrierten WissensmanagementModells vereinen lassen. Als konsequent durchzuhaltender Optimierungsmaßstab hat sich gerade für virtuelle Organisationen die Orientierung an größtmöglicher Flexibilität bezüglich Maßnahmen und Technologien zur Unterstützung der 
Selbstorganisation und an der Berücksichtigung der Dynamik individueller Kosten-Nutzen-Bilanzen bewährt. Der hier vorgestellte Etablierungsprozess hat den Vorteil, von Beginn an ein positives Kosten-Nutzen-Verhältnis aufzuweisen und so die Hemmschwelle zu einer aktiven Beteiligung herabzusetzen. Der Schwerpunkt liegt auf einer IuK-Technik-basierten Kommunikation, wobei großer Gestaltungsspielraum für unterstützende Werkzeuge, die im Konzept zum Einsatz kommen, besteht.

Auch wenn es gelungen ist, einige Anforderungen an ein Zielsystem zur Unterstützung für Knowledge Communities aus den Erfahrungen mit der Praxis der virtuellen Organisationen herauszufiltern, können wir weder den Anspruch erheben, eine komplette Anforderungsbeschreibung liefern zu können, noch haben wir bisher ein System gefunden, welches hinsichtlich Flexibilität und Selbstorganisationsunterstützung den beschriebenen Ansprüchen genügen würde. Hier ist noch Forschungs- und Umsetzungsbedarf. Unsere Orientierung an einem Prozessmodell zielte auch darauf ab, mit existierenden Technologien das Mögliche zu erreichen.

Knowledge Communities eignen sich für ein Wissensmanagement in virtuellen Organisationen, da dort aufgrund ihrer charakteristischen Eigenschaften ein gemeinsames Vertrauensverständnis aller Beteiligten untereinander erschwert wird. Communities - dies zeigt sich auch in anderen Bereichen - benötigen und sind gleichzeitig geeignet, eine vertrauensvolle Kultur zu schaffen, in der Wissensaustausch und gemeinsame Wissensgenese für alle Beteiligten gewinnbringend gelebt werden können.

\section{Danksagungen}

Wir bedanken uns bei Bettina Törpel, Wolfgang von Berg, Volker Wulf und Ralf Zimmermann für wertvolle Diskussionen zum Thema und tatkräftige Mitarbeit bei den Forschungsarbeiten. Die Forschungsarbeiten wurden im Rahmen des Projektes "Olvio - Organisationales Lernen in Virtuellen Organisationen" durch das BMB+F gefördert (Fö.-Kz. 01HG8894).

\section{Literatur}

[AcHa98] Ackerman, M.S., Halverson, C. (1998): Considering an Organization's Memory. in Int. Conf. on CSCW'98, (Seattle, WA, 1998), ACM Press, 39-48

[AcMc96] Ackerman, M.S., McDonald, D.W. (1996): Answer Garden 2: Merging Organizational Memory with Collaborative Help", in: International Conference on CSCW'96, Boston, MA, ACM-Press, 97-105 
[Aks ${ }^{+} 98$ ] Akscyn, R.M.; McCracken, D.L.; Yoder, E.A. (1988): KMS: A distributed Hypermedia system for managing Knowledge in Organizations. Communications of the ACM, 31 (7). 820-835.

[ArHä95] Arnold O., Härtling M., Virtuelle Unternehmen: Begriffsbildung und -diskussion, Arbeitspapier der Reihe Informations- und Kommunikationssysteme als Gestaltungselement virtueller Unternehmen, Bern, 1995

[BaKu96] Bannon, L.; Kuutti, K. (1996): Shifting Perspectives on Organizational Memory: From Storage to Active Remembering, in: Proceedings of HICSS-29, 156-167

[BYRN99] Baeza-Yates, R., Ribeiro-Neto, B.: Modern Information Retrieval, Addison Wesley, 1999, 464 Seiten

[BrDu00] Brown, J.S. und Duguid, P.: Organizational Learning and Communities of Practice: Towards a Unified View of Working, Learning, and Innovation, in: Lesser, E.L., Fontaine, M.A., Slusher, J.A.: Knowledge and Communities, Butterworth-Heinemann, 2000, Woburn, MA

[Bul' ${ }^{+}$97] Bullinger, H.-J., Fähnrich, K.-P., Meiren, T., Groh, G. (1997): „Die Entwicklung der Dienstleistung zum Produkt“, in: Computerworld, 51/97: 7-11.

[Hand95] Handy, C: Trust and the virtual organisation. In: Harvard Business Review, Vol. May-June 1995: S. 40-50.

[Klöc02] Klöckner, K.: BSCW: Cooperation Support for Distributed Workgroups. in: Proceedings of the 10th Euromicro Workshop on Parallel, Distributed and Network-based Processing, 2002, Vajda, S. 277-282.

[Kreb98] Krebs M., Die virtuelle Unternehmung als Wissensorganisation: Potentiale und Grenzen des Wissensmanagements, Arbeitspapiere des FB Wirtschaftwissenschaften, Universität Wuppertal, Nr. 181, 1998

[Lees97] Lees, D.Y.: An Analysis of Knowledge Work and its Implications for the Design of Information Artefacts. Faculty of Computer Studies and Mathematics, University of the West of England, Bristol, UK, 1997

[Les $\left.{ }^{+} 00\right]$ Lesser, E.L., Fontaine, M.A., Slusher, J.A.: Preface, in: Lesser, E.L., Fontaine, M.A., Slusher, J.A (Hrsg.): Knowledge and Communities, Butterworth-Heinemann, 2000, Woburn, MA.

[Mo97] Mowshowitz, A. Virtual Organization. Communications of the ACM, 40 (9). 3037.

[NoTa95] Nonaka, I.; Takeuchi, H. (1995): The Knowledge-Creating Company. Oxford University Press, New York, USA, 1995.

[NoPr00] Nonnecke, B.; Preece, J.: Lurker Demographics: Counting the silence. In: Proceedings of CHI 2000, Den Haag, Niederlande, ACM Press, S. 73-80

[Orli00] Orlikowski, W. J.: Using Technology and Constituting Structures: A Practise Lens for Studying Technology in Organizations, in: Organization Science, Vol. 11, No. 4, 2000, pp. $404-428$ 
[Orr90] Orr, J. (1990): Sharing Knowledge, Celebrating Identity: War Stories and Community Memory in a Service Culture; in: Middleton, D.S., Edwards, D. (Hrsg.): Collective Remembering: Memories in Society, Sage Publ., Beverly Hills, CA; USA

[Pi $\left.{ }^{+} 98\right]$ Picot, A., Reichwald, R., Wigant, R.: „Die grenzenlose Unternehmung - Information, Organisation und Management“, 3. Aufl., Gabler, Wiesbaden, 1998.

[PiWo02] Pipek, V., Won, M.: Communication-oriented Computer Support for Knowledge Management. Informatik/Informatique - Magazine of the Swiss Informatics Societies 2002(1): 39-43.

[Pipe ${ }^{+}$03a] Pipek, V., Nuderscher, P., Won, M.: Periphere Wahrnehmung von Expertise, in: Mambrey, P., Pipek, V., Rohde, M. (Hrsg.): Wissen und Lernen in Virtuellen Organisationen, Physica, Heidelberg, 2003, S. 161-180

[Pipe ${ }^{+}$03b] Pipek, V.; Hinrichs, J.; Wulf, V. (2002): Sharing Expertise: Challenges for Technical Support, in: in: Ackerman, M.; Pipek, V.; Wulf, V. (eds): Expertise Sharing: Beyond Knowledge Management, MIT-Press, Cambridge MA (in press)

[Ri $\left.{ }^{+} 98\right]$ Rittenbruch M., Kahler H., Cremers A. B., Supporting Cooperation in a Virtual Organization, 19th Annual International Conference on Information Systems, Helsinki, Finland, 1998

[Shum97] Shum, S. B.: Negotiating the Construction and Reconstruction of Organisational Memories; in: Journal of Universal Computer Science (Special Issue on IT for Knowledge Management), 3 (8), 1997, 899-928, http://www.jucs.org/

[SuSh98] Sumner, T.; Shum, S. B.: From Documents to Discourse: Shifting Conceptions of Scholarly Publishing. in CHI 1998: Human Factors in Computing Systems, (Los Angeles, CA, USA, 1998), ACM Press, New York, S. 95-102.

[Seng01] Senge, M.: Die fünfte Disziplin. Kunst und Praxis der lernenden Organisation, Klett-Cotta, 2001

[Tör $\left.{ }^{+} 03\right]$ Törpel, B.; Pipek, V.; Rittenbruch, M.: Creating Heterogeneity - Evolving Use of Groupware in a Network of Freelancers, Computer Supported Cooperative Work: The Journal of Collaborative Computing (JCSCW), Vol. 12, No. 1-2, 2003

[WaUn91] Walsh, J.P.; Unger, G.R. (1991): Organizational Memory. Academy of Management Re-view, 16 (1). 57-91.

[Weng98] Wenger, E.: Communities of Practice - Learning, Meaning and Identity. Cambridge University Press, 1998, Cambridge, MA 